\title{
Entamer des conversations d'une importance capitale sans rompre les ponts
}

\author{
par Shirin Abadi
}

$\mathrm{Au}$ fil des ans, j’ai rencontré plusieurs situations au cours desquelles j’ai observé que des gens, moi y compris, hésitaient à faire part de points de vue importants, car ils s'inquiétaient de la réception de ceux-ci. Il est plus facile d'être d'accord avec les personnes qui nous entourent que d'offrir un point de vue différent, en particulier si nous nous entretenons avec des cadres supérieurs ou des personnes ayant plus d'expérience que nous. Alors, comment doit-on aborder ces situations? Comment entamer des conversations qui revêtent une importance capitale sans rompre les ponts?

Dans certains environnements d'affaires, si vous ne proposez pas de point de vue différent pendant une réunion, vos collègues vous dévalorisent. Les gens s'attendent à obtenir des points de vue différents, qui ne sont pas traités comme des exceptions, et ils n'apprécient guère des raisons comme " On a toujours fait comme ça. " Ils ne préconisent pas la réflexion de groupe, mais ils accueillent favorablement les séances de remue-méninges. En fait, souvent, c'est ainsi que naît l'innovation : en réfléchissant aux idées impensables. Vous savez? Celles qu'on accueille en levant les yeux au plafond?

Mais on pourrait dire que l'environnement des soins de santé est un milieu différent, peu enclin à prendre des risques, qui met une insistance particulière sur la sécurité du patient. Nos options de travail avec les partenaires stratégiques sont limitées, aussi ne voulons-nous pas risquer de rompre nos liens avec eux. Le monde de la pharmacie clinique est relativement restreint, c'est pourquoi, nous ne souhaitons pas que nos paroles ou nos actes se retournent contre nous. Qu'est-ce que je dis face à ces arguments? Le « comment » est aussi important que le « quoi »! Le contenu est important, mais la manière de s'exprimer ou d'aborder une situation est tout aussi importante, sinon plus.
Permettez-moi d'expliquer cela plus en détail en vous indiquant une approche toute simple pour exprimer votre point de vue « radical» :

1. Déterminer l'objectif commun de la discussion. Quel fil conducteur rassemble les intervenants? Quelle est la mission du projet ou la vision sous-jacente, quel est le travail de la commission ou celui du comité, etc.?

2. Cerner le problème que chacun essaie de résoudre. Tout le monde peut-il s'accorder sur ce point?

3. Explorer les options qui peuvent être retenues. Il s'agit d'un aspect crucial du processus, où les gens doivent écouter sans tirer de conclusions hâtives. C'est la phase du remue-méninges. Inviter chacun à creuser et à noter des idées. Créer un espace sécurisant, où l'on accueille une diversité de points de vue.

4. Examiner les avantages et inconvénients de chaque option. Cela peut inclure une analyse des coûts et des recettes, une étude de la sensibilité aux risques ainsi que des considérations budgétaires. Plus votre analyse est approfondie, plus les fondements de votre décision finale seront solides.

5. Prenez votre décision définitive en vous basant sur les étapes 1 à 4.

Ce processus vous dit-il quelque chose? Eh bien, il ressemble à celui que nous utilisons pour déterminer et résoudre les problèmes liés à la pharmacothérapie, où chaque étape d'une communication efficace est empreinte de transparence et de respect. En adoptant une approche systématique pour faciliter les conversations d'une importance capitale, nous pouvons détourner l'attention de la personne qui offre un point de vue différent et nous focaliser sur l'aspect le plus nécessaire de la proposition à faire valoir, soit résoudre un problème important.

[Traduction par l'éditeur]

Shirin Abadi, B. Sc. (Pharm.), ACPR, Pharm. D., DPLA, MBA, FCSHP, RPh, est présidente élue et agente de liaison externe pour la Société canadienne des pharmaciens d'hôpitaux. 